\title{
Conscious and subconscious arm movements: Application of signal detection theory to motor control
}

\author{
ANDREW M. GORDON and DAVID A. ROSENBAUM \\ Hampshire College, Amherst, Massachusetts
}

\begin{abstract}
People sometimes carry out voluntary movements that do not seem to be consciously controlled. A familiar example is the board game Ouija. We hypothesize that movement speed is a primary determinant of whether a movement is conscious or subconscious. In support of this hypothesis, we show that mean movement velocity is slower when subjects imagine "a force pushing against the arm" than when they move the arm "as slowly as possible." We also show that this outcome can be accounted for with signal detection theory, a theoretical tool that has so far been used primarily in the areas of perception and memory.
\end{abstract}

Athletic coaches sometimes make use of a diagram like that shown in Figure 1 to demonstrate the "power of thought" in performance. The trainee is told to suspend a weighted string directly over the center of the cross, using the thumb and forefinger, and to imagine that the weight moves along the vertical line of the cross. The weight is often observed to do so. When the trainee is again told to keep the hand perfectly still but now to imagine that the weight moves along the horizontal line of the cross, it can be observed to swing to and fro in this direction. Finally, if the instruction is to imagine that the weight moves circularly, again it can be seen to follow the designated path. The point of such demonstrations is that thinking something can make it happen; thinking of swimming fast, for example, can actually result in fast swimming.

In this paper we are less concerned with the efficacy of this training technique than with the mechanisms of motor control that it relies on. How is it possible to move one's hand voluntarily without being aware of doing so? It is well known that people can be trained with biofeedback to carry out movements they never realized they could (see, e.g., Basmajian, 1963). What is less clear is how people can voluntarily carry out movements they do not know they are making. We seek in this paper to resolve this apparent paradox.

This research was originally conducted by the first author in fulfillment of the requirements for a Division I examination in the School of Communications and Cognitive Science, Hampshire College. The results were also reported by the first author at the 36th Annual Psychology and Education Conference, Mount Holyoke College, April 23, 1983. Preparation of this paper was supported in part by Grant BNS-8120104 from the National Science Foundation to the second author. We thank Ellis Mayers, whose ingenious coaching methods inspired the investigation of this phenomenon. Requests for reprints should be sent to David A. Rosenbaum, School of Communications and Cognitive Science, Hampshire College, Amherst, MA 01002.

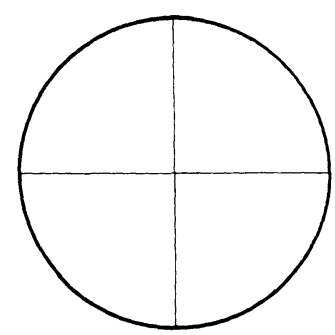

Figure 1. Diagram used to demonstrate the "power of thought" in performance.

The coaching device described above has been used at least since the early 19th century, when Chevreul (see Delpratto, 1977) announced the discovery of a new branch of physics in which psychic energy could be harnessed and scientifically studied with what has come to be called Chevreul's pendulum (Delpratto, 1977; Jastrow, 1937). Chevreul's pendulum is familiar to many people in the form of the board game Ouija. Here the player places one hand on a platform that can slide with little friction from one place to another on a board with different items of personal interest. The player is asked a question and then "waits to see where the platform goes," ostensibly under the influence of an external (spiritual) force. A less esoteric phenomenon has been reported recently by Kowler and Steinman (1978). They noted that when human subjects expect a visual target to move in a particular direction, the eyes exhibit a slow drift in the direction of the expected motion. Once again, the eye movement occurs without conscious awareness.

In the present study, we tested the hypothesis that movement speed is the main determinant of whether an executed movement is consciously or subconsciously controlled. We propose that movements that are consciously controlled may simply be faster than movements that are subconsciously controlled. We tested 
this hypothesis by measuring the speed of movement produced under instructions that were meant to induce either a conscious or a subconscious movement set. The results unambiguously support our hypothesis. We also provide an explanation of our findings that uses the constructs of signal detection theory (Green \& Swets, 1966).

\section{METHOD}

\section{Procedure}

The movements tested were adductions of the extended right arm in the horizontal plane. The subject began each trial by holding his or her right arm straight ahead (i.e., at an angle of $90 \mathrm{deg}$ with respect to the frontal plane of the body) and in the shoulder plane. Movement of the arm was recorded by using a strip-chart recorder, in a manner described below.

There were three conditions. In the control condition the subject was instructed to hold the arm as still as possible. In the "conscious movement" condition the subject was instructed to move the arm as slowly and as steadily as possible toward the left. In the "subconscious movement" condition the subject was instructed to imagine a force continually pushing the arm toward the left.

The three conditions were administered in a counterbalanced order to the 12 subjects in the experiment. Each subject was tested in each condition in a single $50-\mathrm{sec}$ trial.

\section{Materials}

The test movement required the use of the deltoid and pectoralis muscles, as well as the shoulder joint. To restrict all other movement, a yardstick was secured to the underside of the subject's right arm, extending from the armpit to approximately $1 \mathrm{ft}$ beyond the fingertips. The yardstick prevented movement about the elbow joint or wrist and isolated movement to the shoulder joint. At the end of the yardstick, a marker was attached at a 45-deg angle; the marker was used to record movement on the graph of a strip-chart recorder (Grass Instruments Model H330-P, paper speed $=15 \mathrm{~mm} / \mathrm{sec}$ ).

The subject was seated in an adjustable chair so that when both arms were extended forward, the marker rested on the graph paper and the left hand rested on a platform mounted on the strip-chart recorder.

The subjects were blindfolded at all times.

\section{Measurement}

To determine the mean velocity of the arm movement, the distance between the starting point and the finishing point on the graph was measured to the nearest millimeter. This distance was then divided by the duration of the trial, measured to the nearest second. The resulting quotient of distance divided by time served as the estimate of the mean velocity of the arm movement performed in the trial.

In addition to estimating the mean velocity in this way, the graphs were also scrutinized for deviations from the principal trajectories. In the experimental conditions, two types of deviations were sought: (1) flat lines, indicating no movement; and (2) lines sloping in the direction opposite to the principal trajectory, indicating backtracking. In the control condition, the indices of deviation were the number of line segments veering away from the starting position and the amplitude of these line segments.

\section{Subjects}

Twelve Hampshire College students, seven males and five females, volunteered to be subjects. All the subjects were righthanded and had normal sensorimotor capabilities.
Table 1

Mean Velocity of Movement (in Millimeters per Second)

\begin{tabular}{ccc}
\hline & \multicolumn{2}{c}{ Condition } \\
\cline { 2 - 3 } Subject & Conscious & Subconscious \\
\hline 1 & 2.14 & .97 \\
2 & 4.00 & 1.16 \\
3 & 3.50 & 1.08 \\
4 & 10.37 & 2.98 \\
5 & 2.27 & 2.20 \\
6 & 2.45 & 1.29 \\
7 & 5.31 & 1.96 \\
8 & 3.90 & .74 \\
9 & 1.98 & 1.36 \\
10 & 1.09 & .81 \\
11 & 8.00 & 3.85 \\
12 & 2.92 & 1.11 \\
Mean & 3.99 & 1.63 \\
\hline
\end{tabular}

\section{RESULTS}

The mean movement velocities for all 12 subjects in the conscious and subconscious movement conditions are listed in Table 1. For every subject the mean velocity of movement was higher in the conscious movement condition than in the subconscious movement condition. The difference between movement velocities in these two conditions was highly significant, as tested with the related-groups $\mathrm{t}$ test $[\mathrm{t}(11)=-1.96, \mathrm{p}<$ .005 ]

In the control condition, the right arm exhibited a series of small random-direction excursions. The maximum deviation from the starting position, averaged over subjects, was $11 \mathrm{~mm}$. This value can be compared with the displacements of 82 and $200 \mathrm{~mm}$ obtained in the subconscious movement and conscious movement conditions, respectively.

In analyzing the velocities from the two experimental conditions, we noted a somewhat unexpected result: Those subjects who had high velocities in one experimental condition also had high velocities in the other. Across subjects, the correlation between velocities in the two experimental conditions was .79 , and the slope and zero-intercept of the best-fitting straight line fitted to the velocity points obtained by plotting conscious movement velocity against unconscious movement velocity were $2.24 \mathrm{~mm} / \mathrm{sec}$ and $.35 \mathrm{~mm} / \mathrm{sec}$, respectively. The latter result provides a basis for a model that can account for the main features of our results. The model is presented in the next section.

\section{DISCUSSION}

The results of this experiment can be accounted for with signal detection theory (Green \& Swets, 1966). Consider the curves in Figure 2. The leftmost curve represents the distribution of excitation assumed to emanate from proprioceptors of the arm when the arm is stationary. The two curves on the right represent distributions of excitation emanating from proprioceptors 


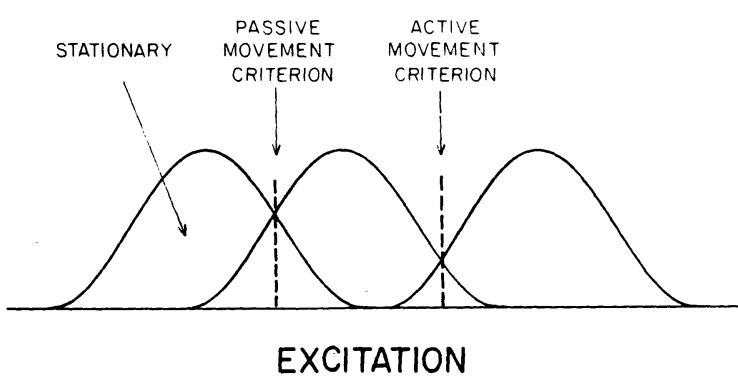

Figure 2. Distributions of proprioceptive excitation assumed to arrive at a central decision site when the arm is stationary or in motion, and criteria assumed to be used in determining whether the arm is in motion under conscious control (active movement criterion) or under subconscious control (passive movement criterion).

of the arm when the arm is in motion. Suppose the subject determines whether his or her arm is moving by deciding whether the proprioceptive excitation arriving at a central decision site exceeds a criterion. Suppose, moreover, that the criterion is more rigorous (i.e., farther to the right on a scale like that shown in Figure 2) in the conscious movement condition than in the subconscious movement condition. Finally, suppose that the level of proprioceptive excitation coming to the central decision site is proportional to the velocity of arm movement-an assumption supported by physiological data (Body \& Roberts, 1953; see also Adams, 1977, for review).

With these assumptions, we can account for our results. The fact that subjects moved their arms at a higher velocity in the conscious movement condition than in the subconscious movement condition is explained by saying that a higher level of proprioceptive excitation had to be produced in the conscious movement condition. The way this higher level of excitation was produced was to increase the velocity of movement. As for the correlation between conscious and subconscious movement velocities obtained across subjects, this outcome can be explained by adding the assumption that different subjects had different baseline excitation levels and, therefore, that higher absolute levels of excitation were required for some subjects than for others.

We believe this explanation of our data is important for two reasons. First, the explanation helps to clarify a hitherto mystifying phenomenon of human performance: What has sometimes been considered a reflection of the metaphysical can, with our model, be understood in terms of the psychophysical. The second reason for attaching importance to our model is that it comprises one of the first applications of signal detection theory to motor control that we know of.

\section{REFERENCES}

Adams, J. A. (1977). Feedback theory of how joint receptors regulate the timing and positioning of a limb. Psychological Review, 84, 504-523.

Basmajian, J. V. (1963). Control and training of individual motor units. Science, 141, 440-441.

Boyd, I. A., \& Roberts, T. D. M. (1953). Proprioceptive discharge from stretch-receptors in the knee joint receptors of the cat. Journal of Physiology, 122, 38-58.

Delpratto, D. J. (1977). Observing covert behavior with Chevreul's pendulum. Psychological Record, 2, 473-478.

Green, D. M., \& Swets, J. A. (1966). Signal detection theory and psychophysics. New York: Wiley.

JASTROW, J. (1937). Chevreul as psychologist. Scientific Monthly, 44, 487-496.

Kowler, E., \& Steinman, R. (1978). The effects of expectations on slow oculomotor control-II. Single target displacements. Vision Research, 19, 633-646.

(Manuscript received for publication December 27, 1983). 